\title{
Progress in Competing Endogenous RNA and Cancer*
}

\author{
Ting Zhang1, Weiguo Huang1,2\# \\ ${ }^{1}$ Cancer Research Institute, University of South China, Hengyang, China \\ ${ }^{2}$ Post-Doctoral Mobile Stations of Preclinical Medicine, University of South China, Hengyang, China \\ Email: " hwg doctor@126.com
}

Received 26 June 2015; accepted 24 July 2015; published 27 July 2015

Copyright (C) 2015 by authors and Scientific Research Publishing Inc.

This work is licensed under the Creative Commons Attribution International License (CC BY).

http://creativecommons.org/licenses/by/4.0/

(c) (i) Open Access

\section{Abstract}

The competing endogenous RNA (ceRNA) hypothesis was introduced as a previously unrecognized gene regulatory layer. This is a recently discovered hypothesis about how mRNAs, pseudogene transcripts, long noncoding RNAs (IncRNAs) and circular RNAs (circRNAs) regulate post-transcriptional expression by sharing common microRNA response elements (MREs) to compete for the binding of microRNAs (miRNAs) and inhibiting normal miRNA targeting activity on mRNA. Previous study have found that ceRNAs are key regulators in many biological processes such as cell cycle, tumor initiation and progression and found to be involved in several diseases, especially in tumor. Tumor is a kind of serious disease with high death rate. Recently, there is no effective therapy for Tumor. The mechanism of many tumors has not yet been fully elucidated. It has been proved that a number of ceRNAs identified as aberrantly expressed during tumor development. It is vital to understand how ceRNA works in tumor progression and to find a new way to cure those corresponding disease. This review introduces the mechanism of ceRNA hypothesis, focus on the history of the ceRNA hypothesis, the important pathophysiological roles of ceRNAs in tumor and the latest findings about how ceRNA works in gastric cancer, lung cancer, endometrial cancer, breast cancer, liver cancer, colorectal cancer and melanoma.

\section{Keywords}

ceRNA, miRNA, Tumorigenesis, miRNA and mRNA Interaction

\section{Introduction of ceRNA}

Pandolfi and his research groups are scientists from Harvard Medical School. They issued a new hypothesis [1]

${ }^{*}$ This project was supported by the Chinese National Science foundation (No. 81172210) and China Postdoctoral Science Foundation (No. 2012M521528).

"Corresponding author. 
about how long noncoding RNAs and mRNA "talk" to each other and this hypothesis is named as ceRNAs. This is the first time we proposed ceRNA hypothesis. The biological function of ceRNAs is done through sharing common MRE compete for the binding of same miRNA. miRNA and MRE are regarded as two important elements of the ceRNA hypothesis. miRNAs are a class of endogenous, non-coding 20 - 23 nucleotides RNA molecules. They perform post-transcriptional regulation by specifically binding target messenger RNAs (mRNAs) through combination with MRE and resulting in a reduction in the levels of the corresponding proteins [2]-[4]. Each mRNAs contains multiple MREs and thus can be regulated by a number of miRNAs, while one miRNA can potentially regulate dozens of mRNA transcripts [3] [5]. Increasing experimental evidences support the hypothesis that multiple non-coding RNA species, including small non-coding RNAs, pseudogenes, lncRNAs and circRNAs can act as miRNA "sponges" by sharing identical MREs compete for common miRNAs and inhibiting normal miRNA activity [6] [7]. These sponges "absorb" miRNAs, lowering the levels of available miRNAs for the target mRNA, resulting in increased translations. The first endogenous "sponge" RNA was discovered in plants in a situation where a stress related miRNA-mediated response was reduced [8]. In October 2011, research institutes from Harvard Medical School, Columbia University and the University of Roma La Sapienza published four research results [9]-[12] on the Cell. They verified Pandolfi's hypothesis about ceRNA from different aspects and further confirmed the existence of ceRNAs regulation mechanism. The discovery of the Mechanism of ceRNAs has subverted the traditional understanding of the function of mRNA. The function of messenger RNA (mRNA) is to not only deliver protein-coding information to sites of protein synthesis but also participate in the regulation of gene expression. CeRNAs cross-regulate each other through sequestration of shared miRNAs and that this "competing endogenous RNA" activity forms a large-scale regulatory network across the transcriptome, greatly expanding the functional genetic information in the human genome.

The ceRNAs play a crucial role in many biological processes and the destruction of the balance between ceRNAs and miRNAs can be important for ceRNA activity and contributing a lot to diseases like cancer. It is reported that ceRNAs was a very important regulator in many types of cancer. ceRNAs have also been found to be involved in cancer developmental stages. In the following part of the review, we will concentrate on formation process of ceRNA hypothesis and its relationship with cancer.

\section{The History of ceRNA Hypothesis}

In fact, back to 2007, the concept of competitive RNA has already existed. Until 2011, scientists proposed formally this hypothesis. At the year 2007, Ebert [13] constructed a miRNA inhibitor called miRNA sponge, these competitive inhibitors are transcripts expressed from strong promoters, containing multiple, tandem binding sites to a microRNA of interest. When vectors encoding these sponges are transiently transfected into cultured cells, sponges derepress microRNA targets. By competitive binding specific mRNA, miRNA sponge can block the interaction between miRNA and its natural target, thereby inhibiting endogenous miRNA function.

In 2009, Seitz [14] proposed pseudotarget hypothesis. The main idea of the hypothesis is that nature and artificial miRNA pseudotarget actually be competitive inhibitors of miRNA, prevent miRNAs from binding their authentic targets by sequestering them. The hypothesis also predicts that the decrease of miRNA binding with pseudotargets would increase the concentration of free miRNA, hence enhancing the repression of authentic targets.

In 2010, Arvey [15] proposed a dilution effect (dilutioneffect) hypothesis. They hypothesize that an intracellular pool of miRNAs/siRNAs faced with a larger number of available predicted target transcripts will downregulate each individual target gene to a lesser extent.

In 2010, Ebert [16] proposed a "natural miRNA sponge" concept. Based on the existing experimental evidence, they think, there are natural miRNA sponge exists in cells, these endogenous miRNA sponge (including non coding RNA) block miRNA activity through the combination with specific target mRNA and influence the expression of miRNA target genes at the post transcriptional level. On the basis of these experimental findings [6] [17] [18], it was hypothesized that a myriad of natural sponges may exist and play critical roles in biologic processes.

In August 2011, Salmena [1] proposed the ceRNA hypothesis formally. Different from exogenous miRNA sponge, ceRNA is endogenous RNA including messenger RNAs, transcribed pseudogenes, and long noncoding RNAs. They "talk" to each other using MREs as letters of a new language. A ceRNA chain may contain multiple MRE, can be combined with different miRNA, and each miRNA can silence multiple transcripts, thus forming a large-scale complex regulatory network across the transcriptome. 


\section{The Latest Research Progress of ceRNA Hypothesis}

As time goes by, scientists have made a further research of ceRNA and we had a deeper understanding of the hypothesis of ceRNA. Some researchers have put forward that ceRNAs form complex regulatory networks and cross-regulate each other through sequestration of shared microRNAs [19]. Ugo Ala et al. [19] had established a mode to verified that when given optimal molecular conditions, alterations of one single ceRNA can have significant effects on transcriptional networks and integrated ceRNA. Marcella Cesana et al. [20] also recommended the rules of ceRNA networks. Although the molecular requirements for ceRNA cross-regulation and the extent of ceRNA networks remain unknown, we can draw the conclusion that all the members of ceRNA make up the complex ceRNA net-work. They are closely related to each other and can influence each through the net-work.

\section{The Important Pathophysiological Roles of ceRNAs in Cancer}

As mentioned above, scientists have put forward the concepts of miRNA sponge, pseudotarget hypothesis, dilution effect hypothesis, natural miRNA sponge and ceRNA net-works. Those concepts are basis for ceRNA hypothesis. Based on those concepts and the research results from other scientists, Salmena proposed ceRNA hypothesis. As time goes by, advances in experimental techniques allowing us to further study this hypothesis. More and more evidences have been found to prove the existence of ceRNA hypothesis. The experimental evidence make people firmly believe that ceRNAs plays a vital role in biology process and in the progress of disease, partically in tumorigenesis. ceRNAs, oncogenes, anti-oncogenes and target gene 3'-UTR of tumor related signaling pathway which shared common MRE can competive for binding of the same miRNA. Thus ceRNAs can regulate miRNA's function as oncogenes or tumor suppressor genes and are corresponding to cell proliferation, apoptosis, invasion and metastasis and drug resistance. Here we will give some examples on how ceRNA works in some cancers according to the latest study.

\subsection{Gastric Cancer}

Gastric cancer happens at a high rate among gastrointestinal malignancy with worldwide prevalence and the second leading cause of cancer related death. Although much progress has been made in recent years, most of patients with gastric cancer are diagnosed at an advanced stage and accompanied by malignant proliferation, extensive invasion and lymphatic metastasis. Successful prognosis method and therapeutic strategies are limited, thus the mortality is high [21] [22]. Recent studies indicate that long non-coding RNA is a crucial member of ceRNA networks that harbors MRE and linking miRNAS to regulate gene expression at post-transcriptional network. The latest study reveals that this mechanism is also works in gastric cancer progression and metastasis. HER2 (human epithelial growth factor receptor 2) is one of the target of miR-331-3p and it can encode a transmembrane protein. This kind of protein is very important in carcinogenesis and resistance to trastuzumab-based therapy [23]. miR-331-3p can prevent the occurrence of gastric cancer by inhibite HER2 expression levels through combination with its MRE. HOTAIR (Hox transcript antisense intergenic RNA) is a 2.2-kb long non-coding RNA transcribed from the HOXC locus, which can repress transcription in trans of HOXD in foreskin fibroblasts [24]. In Liu's [25] receach, he reveals that Lnc RNA HOTAIR functions as a competing endogenous RNA to regulate human epithelial growth factor receptor 2 (HER2) expression through competitive for the binding of miR-331-3p and abolishing the miRNA-induced repressing activity on the HER2 3'-UTR, thus playing an oncogenic role in gastric pathogenesis. HOTAIR was upregulated in gastric carcinoma tissues compared with normal gastric tissues. The upregulation of HOTAIR result in larger tumor size, advanced pathological stage and extensive metastasis. HOTAIR function as a ceRNA is a new research directions and therapeutic options in gastric cancer. Liu et al.'s research indicates that the ceRNA activity of HOTAIR imparts a miRNA/lncRNA trans-regulatory function to protein-coding mRNAs and the ceRNA network may play an important role in gastric pathogenesis.

\subsection{Lung Cancer}

Lung cancer is one of the most prevalent cancer worldwide. It is vital to understand the basis for lung cancer progression. Many studies found that ceRNA network have important functional role in the progression of lung cancer. A recent study by Kumar [26] show that Hmga2 (High Mobility Group A2) contributes to lung cancer 
progression and metastasis by operating as a ceRNA for the let-7 miRNA family. The former study show that Hmga2 as a non-histone chromosomal high mobility group protein plays a important role in promoting lung cancer progression and metastasis. But in the author's research, he found that Hmga2 promotes lung carcinogenesis as both a protein-coding gene and a non-coding gene. The Hmga2 3'UTR contains seven conserved MREs for the let-7 family microRNAs [27], which has previously been demonstrated to constrain lung cancer development [28]. Kumar et al. proved that when inhibit Hmga2's function as a proten-coding gene, it can also promote lung carcinogenesis by providing conserved MRES for let7-family and acting as ceRNAs. Kumar et al. have also indicated that TGF- $\beta$ co-receptor Tgfbr3 is the target gene of Hmga2. Let-7 family is a tumor-surpressor gene which can suppress the expression of Tgfbr3, thus prevent TGF- $\beta$ signaling and result in inhibiting of the growth of tumor. High expression of Hmga2 in lung cancer corresponding with up-regulation of Tgfbr3 via function as a ceRNA for the let-7 family. As a result up-regulation of Tgfbr3 will promote TGF- $\beta$ signalling and result in tumor progression.

The conclusion of Kumar's research is that except for acting as a protein-coding gene, Hmga2 also can decoy the let-7 miRNA family to regulate Tgfbr3 expression and enhances TGF- $\beta$ signaling, thereby promotes lung cancer progression. Kumar's research results are different from the previous study. It contributes a lot to discover the mechanism of lung cancer and gives a new point to find out a new therapy for this worldwide disease.

\subsection{Endometrial Cancer}

Endometrial Cancer happens in a high rate among women. The mechanisn of this cancer is not clear yet. The recent study found that ceRNA is one of the factor to promote this cancer. Core transcription factors (TFs) have been shown to expressed in human endometrial adenocarcinoma and that the expression of core TFs was important for the pluripotency of ETs [29]. miR-145 [30] have been reported to regulate those core TFs directly. Previous study show that miR-145 can down regulate the expression of TFs, thus result in tumorsphere differentiation and inhibite tumor progression. Zhou et al. [31] found that linc-RNA-RoR acts as a "sponge" against mediation of the differentiation of endometrial cancer stem cells by microRNA-145. As lncRNAs is known as a ceRNA, so it can regulate gene expression at post-transcriptional network. Since large intergenic non-coding ribonucleic acids (linc-RNAs) which transcribed from lots of loci in mammalian genomes are another type of lncRNAs, they can also act as ceRNA and might have an important role in cellular processes and gene regulation [32]. linc-RoR was found to be a new linc-RNA and plays an important role in maintaining the pluripotency of human embryo stem cells (hESCs) by acting as an "endogenous sponge" to inhibit micro-ribonucleic acid (miR-145) mediating hESC differentiation [33] [34]. In Zhou et al.'s research he reported that Linc-RoR act as a ceRNA of miR-145, has an important role during endometrial carcinogenesis. High expression level of linc-RoR can release the repression of miR-145 on TFs, thus maintaining the pluripotent state of endometrial tumorspheres (ETs). On the contrary, ET differentiation followed with increased expression of miR-145. Greater expression of miR-145 result in down-regulation of linc-RoR and core TFs, and decreased colony formation. The effects of miR-145 could be inhibited after the expression level of linc-RoR in ETs increasing. The crucial function ceRNA plays in endometrial cancer further verified the important role it plays in progress of tumor.

\subsection{Breast Cancer}

Breast cancer is the most common female malignant tumor. People pay much attention to the study of its pathogenesis. The recent studies on how ceRNA works in breast cancer have made a great progress. The 3'-UTR of Versican mRNA act as ceRNA, it not only play an important role in liver cancer [35], but also can influence breast cancer cell's growth by competitive binding with miR-136, miR-144 and miR-199a-3p and regulate the expression of their target PTEN and RB1 according to Lee's [36] research. PTEN and RB1 are both tumor surpressor genes, the change of PTEN and RB1 expression levels will influence breast cancer cell growth.

Jeyapalan et al. [37] indicated that the 3'-UTR of CD44 serves as a competitor by binding with miR-216a, miR-330 and miR-608. These miRNAs are inhibitor of CD44 and CDC42. High expression of CD44 3'-UTR would function as a decoy when interacting with these endogenous miRNAs and result in an increase in CD44 and CDC42 protein levels which would inhibits proliferation, colony formation and tumor growth in breast cancer. Jeyapalan's result provides a new method to cure brest cancer by using ceRNA mechanism.

Furthermore, Jue Yang et al. [38] validated that FOXO1 3'UTR can function as a ceRNA in repressing metastasis of breast cancer cells via regulating miR-9 activity. Their research further confirmed the ceRNA plays a 
crucial role in the progression of breast cancer. Scientists have demonstrated that by regulating E-cadherin expression directly and promoting EMT, miR-9 could promote the progression of metastasis in breast cancer [39]. Previous studies have indicated that transcription factors like FOXO1, can bind and regulate the function of miRNA [40]. According to Yang et al.'s research, they found that FOXO1-3'UTR and E-cadherin-3'UTR all can bind to miR-9. And their analyses showed that FOXO1-3'UTR and E-cadherin-3'UTR can competitive binding to miR-9. The binding between FOXO1-3'UTR and miR-9 will release the inhibition of miR-9 on E-cadherin and result in upregulation of E-cadherin protein. They come to the conclusion that FOXO1 3'UTR inhibits the metastases of breast cancer cells via induction the expression of E-cadherin. Yang et al.' research results suggest that FOXO1 3'UTR may function as a ceRNA and inhibit the activity of miR-9 and modulating metastasis of breast cancer cells. The examples gives above make people firmly believe that 3 '-untranslated region may also function as ceRNAs and plays a vital role in cancer progression.

\subsection{Liver Cancer}

Liver cancer is a highly malignant cancer. An increasing number of evidences proved that ceRNA network plays an important role in the initiation and progression of liver cancer, which contributes a lot in finding a new therapy to cure liver cancer. Wang et al. [41] demonstrated that Long non-coding RNA (lncRNA), highly up-regulated in liver cancer (HULC) plays an important role in tumorigenesis. They validated that HULC may act as an endogenous "sponge", which down-regulates miR-372's activities. Down-regulation of miR-372 activities can reduce repression of its target gene, PRKACB, which in turn promote PKA signal pathway and result in tumorigenesis. This is another important example for lncRNA act as ceRNA and promote the progression of cancer. As mentioned above, 3'-untranslated region (3'-UTR) can function as ceRNAs and act as oncogene in some kind of tumor such as liver cancer. Fang [33] have indicated that Versican 3'-untranslated region (3'-UTR) functions as a ceRNA and inducing the development of hepatocellular carcinoma by regulating miRNA activity. They found that versican 3'-UTR could bind to miRNAs like miR-133a, miR-199a, miR-144 and miR-431, so as to regulate the expression level of the target gene of these miRNAs, promoting hepatocellular carcinoma cell proliferation, migration and invasion and inhabit apoptosis.

Recent studies also found that pseudogene functions as ceRNA contribute a lot to the development of liver cancer. Octamer-binding transcription factor-4 (OCT4) is a pleiotropic regulator of gene expression in embryonic stem cells. Recent studies demonstrated that OCT4 participates in the initiation and progression of various malignant tumor, such as germ-cell tumors, bladder cancer and liver cancer [42]-[45]. In Wang et al.'s [46] study about the mechanism of liver cancer, they argued that OCT4-pg4, the pseudogene of OCT4, is abnormally activated in hepatocellular carcinoma (HCC) and both gene transcripts can be directly targeted by miR-145. miR-145 is a tumor-suppressive miRNA which can downregulate OCT4 expression levels and inhibite the occurrence of liver cancer. Wang et al. demonstrated that Pseudogene OCT4-pg4 functions as a natural miRNA sponge to regulate OCT4 expression by competing for miR-145, thus upregulate OCT4 protein level in HCC. The upregulation of OCT4 protein level in HCC result in growth and tumorigenicity of HCC cells. Their rsearch show that OCT4-pg4 plays an oncogenic role in hepatocarcinogenesis.

As is mentioned above, long non-coding RNA, versican 3'-UTR and pseudogene, all can act as ceRNA and function a lot in promoting liver cancer. All of these research results validated the existence of ceRNA networks and the important role it plays in carcinogenesis. The analysis of this mechanism sheds new light on the treatment of human HCC.

\subsection{Melanoma}

Inhibition of PTEN (phosphatase and tensin homolog deleted on chromosome ten) and Loss of PTEN, a tumor suppressor gene regulated by ceRNA activity, frequently occurs in tumor progress. And this mechanism attracted much attention from the scientists who studies the molecular factors of cancer. And it also becomes a typical representation of how ceRNA works in carcinogenesis. Karreth et al. [11] demonstrated that aberrant regulation of PTEN via ceRNAs competitive binding to miRNA contributes to melanoma development. They verified zinc finger E-box binding homeobox 2 (ZEB2) is the ceRNA of PTEN by using a mouse model. Both PTEN and ZBE2 containing common microRNA recognition elements (MREs) for miR-25, miR-92a, miR-181 and miR-200b so that they can competive binding to those miRNAs. They proved that ZEB2 modulates PTEN protein levels by acting as a ceRNA. High expression of ZEB2 result in abundant of PTEN protein levels and 
inhabiting the develop of melanoma. When depletion ZEB2's function as a ceRNA, PTEN protein levels will be downregulated which promoting melanoma. Karreth's research supported the notion that ZEB2 has tumor suppressive activity in melanoma cells and ZEB2 prevent the occurrence of melanoma by function as a ceRNA.

\subsection{Colorectal Cancer}

Colorectal cancer (CRC) is the third most commonly diagnosed cancer in male and the second in female worldwide [47]. As is mentioned before, miRNA is a kind of small non-coding RNAs, regulating target genes via the translational inhibition or mRNA degradation and it is very important in cellular biology and pathophysiology. It has been reported that miRNA play pivotal roles in promoting colorectal cancer [48]-[51]. The inhibition of tumor suppressor gene via regulation of ceRNAs also functions a lot in progression of colorectal cancer. Tay et al.'s [9] research demonstrated that mRNAs of gene vesicle-associated membrane protein-associated protein A (VAPA) and CCR4-NOT transcription complex, subunit 6-like (CNOT6L) are ceRNAs of PTEN. VAPA mRNA regulate expression level of PTEN by competive binding for miR-17, miR-19a, miR-20a, miR-20b, miR-26b, miR-106a and miR-106b. While CNOT6L regulate expression level of PTEN by competive binding for miR-17, miR-19a, miR-19b, miR-20a, miR-20b and miR-106b. Thus antagonize PI3K/AKT signaling, and possess growth and tumor-suppressive properties. Upregulation of VAPA and CNOT6L can compete binding to lots of common miRNA with PTEN and deplete the inhabition of miRNA to PTEN. Which results in the upregulation of PTEN expression level and inhabit tumor progress. While dwonregulation of VAPA and CNOT6L induce more mirRNA binding to PTEN and promote carcinogenesis.

\section{Conclusion and Future Perspective}

As is mentioned above, ceRNA plays a provital role in carcinogenesis, and according to examples given above, we can firmly draw the conclusion that ceRNA hypothesis contribute to cancer proliferation, apoptosis, invasion and metastasis. Although many concrete details of ceRNA mechanism remains unknown and the understanding of ceRNA mechanisms and its consequences are in their infancy, we do find a lot of evidences to prove it. More things need to be done to further explore ceRNAs' function in biological process and disease process. The improvement in experimental method and tools allows us to fully explore ceRNA areo and unexplored pathways in cancer. By using ceRNA hypothesis we can explain more disease processes find new therapies to cure cancer. Future challenges will then be to understand more details about ceRNA networks, why such a regulatory networks exist, what are their concrete functions in biological process and diseases. The most important thing is to take full use of this mechanism to find therapies and diagnosis method to ceRNA related diseases. ceRNA is going to be the key for future studies on post-transcriptional regulation and shed light on further study of mechanism of cancer.

\section{References}

[1] Salmena, L., Poliseno, L., Tay, Y., Kats, L. and Pandolfi, P.P. (2011) A ceRNA Hypothesis: The Rosetta Stone of a Hidden RNA Language? Cell, 146, 353-358. http://dx.doi.org/10.1016/j.cell.2011.07.014

[2] Bartel, D.P. (2004) MicroRNAs: Genomics, Biogenesis, Mechanism, and Function. Cell, 116, 281-297. http://dx.doi.org/10.1016/S0092-8674(04)00045-5

[3] Bartel, D.P. (2009) MicroRNAs: Target Recognition and Regulatory Functions. Cell, 136, 215-233. http://dx.doi.org/10.1016/j.cell.2009.01.002

[4] Chekulaeva, M. and Filipowicz, W. (2009) Mechanisms of miRNA Mediated Post-Transcriptional Regulation in Animal Cells. Current Opinion in Cell Biology, 21, 452-460. http://dx.doi.org/10.1016/j.ceb.2009.04.009

[5] Friedman, R.C., Farh, K.K., Burge, C.B. and Bartel, D.P. (2009) Most Mammalian mRNAs Are Conserved Targets of microRNAs. Genome Research, 19, 92-105. http://dx.doi.org/10.1101/gr.082701.108

[6] Poliseno, L., Salmena, L., Zhang, J., Carver, B., Haveman, W.J. and Pandilfi, P.P. (2010) A Coding-Independent Function of Gene and Pseudogene mRNAs Regulates Tumour Biology. Nature, 465, 1033-1038. http://dx.doi.org/10.1038/nature09144

[7] Cesana, M., Cacchiarelli, D., Leqnini, I., Santini, T., Sthandier, O., Chinappi, M., Tramontano, A. and Bozzoni, I. (2011) A Long Noncoding RNA Controls Muscle Differentiation by Functioning as a Competing Endogenous RNA. Cell, 147, 358-369. http://dx.doi.org/10.1016/j.cell.2011.09.028

[8] Ebert, M.S. and Sharp, P.A. (2010) MicroRNA Sponges: Progress and Possibilities. RNA, 16, 2043-2050. 
http://dx.doi.org/10.1261/rna.2414110

[9] Tay, Y., Kats, L., Salmena, L., Weiss, D., Tan, S.M., Ala, U., Karreth, F., Poliseno, L., Provero, P., Di Cunto, F., Lieberman, J., Riqoutsos, I. and Pandolfi, P.P. (2011) Coding-Independent Regulation of the Tumor Suppressor PTEN by Competing Endogenous mRNAs. Cell, 147, 344-357. http://dx.doi.org/10.1016/j.cell.2011.09.029

[10] Sumazin, P., Yang, X., Chiu, H.S., Chung, W.J., Iyer, A., Llobet-Navas, D., Raibhandari, P., Bansal, M., Guarnieri, P., Silva, J. and Califano, A. (2011) An Extensive microRNA-Mediated Network of RNA-RNA Interactions Regulates Established Oncogenic Pathways in Glioblastoma. Cell, 147, 370-381. http://dx.doi.org/10.1016/j.cell.2011.09.041

[11] Karreth, F.A., Tay, Y., Perna, D., Ala, U., Tan, S.M., Rust, A.G., DeNicola, G., Webster, K.A., Weiss, D., PerezMancera, P.A., Krauthammer, M., Halaban, R., Proero, P., Adams, D.J., Tuveson, D.A. and Pandolfi, P.P. (2011) In Vivo Identification of Tumor-Suppressive PTEN ceRNAs in an Oncogenic BRAF-Induced Mouse Model of Melanoma. Cell, 147, 382-395. http://dx.doi.org/10.1016/j.cell.2011.09.032

[12] Cesana, M., Cacchiarelli, D., Leqnini, I., Santini, T., Sthandier, O., Chinappi, M., Tramontano, A. and Bozzoni, I. (2011) A long Noncoding RNA Controls Muscle Differentiation by Functioning as a Competing Endogenous RNA. Cell, 147, 358-369. http://dx.doi.org/10.1016/j.cell.2011.09.028

[13] Ebert, M.S., Neilson, J.R. and Sharp, P.A. (2007) microRNA Sponges: Competitive Inhibitors of Small RNAs in Mammalian Cells. Nature Methods, 4, 721-726. http://dx.doi.org/10.1038/nmeth1079

[14] Seitz, H. (2009) Redefining microRNA Targets. Current Biology, 19, 870-873. http://dx.doi.org/10.1016/j.cub.2009.03.059

[15] Arvey, A., Larsson, E., Sander, C., Leslie, C.S. and Marks, D.S. (2010) Target mRNA Abundance Dilutes microRNA and siRNA Activity. Molecular Systems Biology, 6, 363.

[16] Ebert, M.S. and Sharp, P.A. (2010) Emerging Roles for Natural microRNA Sponges. Current Biology, 20, R858-R861. http://dx.doi.org/10.1016/j.cub.2010.08.052

[17] Franco-Zorrilla, J.M., Valli, A., Todesco, M., Mateos, I., Puga, M.I., Rubio-somozal, I., Levva, A., Weiqel, D., Garcia, J.A. and Paz-Ares, J. (2007) Target Mimicry Provides a New Mechanism for Regulation of microRNA Activity. Nature Genetics, 39, 1033-1037. http://dx.doi.org/10.1038/ng2079

[18] Cazalla, D., Yario, T. and Steitz, J.A. (2010) Down-Regulation of a Host microRNA by a Herpesvirus saimiri Noncoding RNA. Science, 328, 1563-1566. http://dx.doi.org/10.1126/science.1187197

[19] Ala, U., Karreth, F.A., Bosia, C., Pagnani, A., Taulli, R., Leopold, V., Tay, Y., Provero, P., Zechina, R. and Pandolfi, P.P. (2013) Integrated Transcriptional and Competitive Endogenous RNA Networks Are Cross-Regulated in Permissive Molecular Environments. Proceedings of the National Academy of Sciences of the United States of America, 110, 7154-7159. http://dx.doi.org/10.1073/pnas.1222509110

[20] Cesana, M. and Daley, G.Q. (2013) Deciphering the Rules of ceRNA Networks. Proceedings of the National Academy of Sciences of the United States of America, 110, 7112-7113. http://dx.doi.org/10.1073/pnas.1305322110

[21] Coburn, N.G. (2009) Lymph Nodes and Gastric Cancer. Journal of Surgical Oncology, 99, 199-206. http://dx.doi.org/10.1002/jso.21224

[22] Shi, Y. and Zhou, Y. (2010) The Role of Surgery in the Treatment of Gastric Cancer. Journal of Surgical Oncology, 101, 687-692.

[23] Lee, H.E., Park, K.U., Yoo, S.B., Nam, S.K., Park, D, J., Kim, H.H. and Lee, H.S. (2013) Clinical Significance of Intratumoral HER2 Heterogeneity in Gastric Cancer. European Journal of Cancer, 49, 1448-1457. http://dx.doi.org/10.1016/j.ejca.2012.10.018

[24] Rinn, J.L., Kertesz, M., Wang, J.K., Squazzo, S.L., Xu, X., Bruqmann, S.A., Goodnough, L.H., Helms, J.A., Farnham, P.J., Seqal, E. and Chang, H.Y. (2007) Functional Demarcation of Active and Silent Chromatin Domains in Human HOX Loci by Noncoding RNAs. Cell, 129, 1311-1323. http://dx.doi.org/10.1016/j.cell.2007.05.022

[25] Liu, X.H., Sun, M., Nie, F.Q., Ge, Y.B., Zhang, E.B., Yin, D.D., Kong, R., Xia, R., Lu, K.H., Li, J.H., De, W., Wang, K.M. and Wang, Z.X. (2014) Lnc RNA HOTAIR Functions as a Competing Endogenous RNA to Regulate HER2 Expression by Sponging miR-331-3p in Gastric Cancer. Molecular Cancer, 13, 92.

[26] Kumar, M.S., Armenteros-Monterroso, E., Eat, P., Chakravorty, P., Matthews, N., Winslow, M.M. and Downward, J. (2014) HMGA2 Functions as a Competing Endogenous RNA to Promote Lung Cancer Progression. Nature, 505, 212-217. http://dx.doi.org/10.1038/nature12785

[27] Mayr, C., Hemann, M.T. and Bartel, D.P. (2007) Disrupting the Pairing between Let-7 and Hmga2 Enhances Oncogenic Transformation. Science, 315, 1576-1579. http://dx.doi.org/10.1126/science.1137999

[28] Kumar, M.S., Erkeland, S.J., Pester, R.E., Chen, C.Y., Ebert, M.S., Sharp, P.A. and Jacks, T. (2008) Suppression of Non-Small Cell Lung Tumor Development by the Let-7 microRNA Family. Proceedings of the National Academy of Sciences of the United States of America, 105, 3903-3908. http://dx.doi.org/10.1073/pnas.0712321105 
[29] Zhou, X., Zhou, Y.P., Huang, G.R., Gong, B.L., Yang, B., Zhang, D.X., Hu, P. and Xu, S.R. (2011) Expression of the Stem Cell Marker, Nanog, in Human Endometrial Adenocarcinoma. International Journal of Gynecological Pathology, 30, 262-270. http://dx.doi.org/10.1097/PGP.0b013e3182055a1f

[30] Wu, Y., Liu, S., Xin, H., Jiang, J., Younglay, E., Sun, S. and Wang, H. (2011) Up-Regulation of microRNA-145 Promotes Differentiation by Repressing OCT4 in Human Endemetrial Adenocarcinoma Cells. Cancer, 117, 3989-3998. http://dx.doi.org/10.1002/cncr.25944

[31] Zhou, X., Gao, Q., Wang, J., Zhang, X., Liu, K. and Duan, Z. (2014) Linc-RNA-RoR Acts as a “Sponge” against Mediation of the Differentiation of Endometrial Cancer Stem Cells by microRNA-145. Gynecologic Oncology, 133, 333-339. http://dx.doi.org/10.1016/j.ygyno.2014.02.033

[32] Ulitsky, I. and Bartel, D.P. (2013) LincRNAs: Genomics, Evolution, and Mechanisms. Cell, 154, 26-46. http://dx.doi.org/10.1016/j.cell.2013.06.020

[33] Wang, Y., Xu, Z., Jiang, J., Xu, C., Kang, J., Xiao, L., Wu, M., Xiong, J., Guo, X. and Liu, H. (2013) Endogenous miRNA Sponge lincRNA-RoR Regulates Oct4, Nanog, and Sox2 in Human Embryonic Stem Cell Self-Renewal. Developmental Cell, 25, 69-80. http://dx.doi.org/10.1016/j.devcel.2013.03.002

[34] Loewer, S., Cabili, M.N., Guttman, M., Loh, Y.H., Thomas, K., Park, I.H., Garber, M., Curran, M., Onder, T., Agarwal, S., Manos, P.D., DAtta, S., Lander, E.S., Schlaeger, T.M., Daley, G.Q. and Rinn, J.L. (2010) Large Intergenic NonCoding RNA-RoR Modulates Reprogramming of Human Induced Pluripotent Stem Cells. Nature Genetics, 42, 11131117. http://dx.doi.org/10.1038/ng.710

[35] Fang, L., Dum, W.W., Yang, X., Chen, K., Ghanekar, A., Levy, G., Yang, W., Yee, A.J., Lu, W.Y., Xuan, J.W., Gao, Z., Xie, F., He, C., Deng, Z. and Yang, B.B. (2013) Versican 3'-Untranslated Region (3'-UTR) Functions as a ceRNA in Inducing the Development of Hepatocellular Carcinoma by Regulating miRNA Activity. The Journal of the Federation of American Societies for Experimental Biology, 27, 907-919. http://dx.doi.org/10.1096/fj.12-220905

[36] Lee, D.Y., Jeyapalan, Z., Fang, L., Yang, J., Yee, A.Y., Li, M., Du, W.W., Shatseva, T. and Yang, B.B. (2010) Expression of Versican 3'-Untranslated Region Modulates Endogenous microRNA Functions. PLoS ONE, 5, e13599.

[37] Jeyapalan, Z., Deng, Z., Shatseva, Z., Fang, L., He, C. and Yang, B.B. (2011) Expression of CD44 3'-Untranslated Region Regulates Endogenous microRNA Functions in Tumorigenesis and Angiogenesis. Nucleic Acids Research, 39, 3026-3041. http://dx.doi.org/10.1093/nar/gkq1003

[38] Yang, J., Li, T., Gao, C., Lv, X., Liu, K., Song, H., Xing, Y. and Xi, T. (2014) FOXO1 3’UTR Functions as a ceRNA in Repressing the Metastases of Breast Cancer Cells via Regulating miRNA Activity. Federation of European Biochemical Societies, 588, 3218-3224. http://dx.doi.org/10.1016/j.febslet.2014.07.003

[39] Ma, L., Young, J., Prabhala, H., Pan, E., Mestdagh, P., Muth, D., Teruya-Feldstein, J., Reinhardt, F., Onder, T.T., Valastyan, S., Westermann, F., Vandesompele, J. and Weinberg, R.A. (2010) MiR-9, a MYC/MYCN-Activated microRNA, Regulates E-Cadherin and Cancer Metastasis. Nature Cell Biology, 12, 247-56.

[40] Ala, U., Karreth, F.A., Bosia, C., Pagnani, A., Taulli, R., Leopold, V., Tay, Y., Provero, P., Zecchina, R. and Pandolfi, P.P. (2013) Integrated Transcriptional and Competitive Endogenous RNA Networks Are Cross-Regulated in Permissive Molecular Environment. Proceedings of the National Academy of Sciences of the United States of America, 10, 7154-7159. http://dx.doi.org/10.1073/pnas.1222509110

[41] Wang, J., Liu, X., Wu, H., Ni, P., Gu, Z., Qiao, Y., Chen, N., Sun, F. and Fan, Q. (2010) CREB Up-Regulates Long Non-Coding RNA, HULC Expression through Interaction with microRNA-372 in Liver Cancer. Nucleic Acids Research, 38, 5366-5383. http://dx.doi.org/10.1093/nar/gkq285

[42] Yin, X., Li, Y.W., Zhang, B.H., Ren, Z.G., Qiu, S.J., Yi, Y. and Fan, J. (2012) Coexpression of Stemness Factors Oct4 and Nanog Predict Liver Resection. Annals of Surgical Oncology, 19, 2877-2887.

[43] Qian, Y.W., Chen, Y., Yang, W., Fu, J., Cao, J., Ren, Y.B., Zhu, J.J., Su, B., Luo, T., Zhao, X.F., Dai, R.Y., Li, J.J., Sun, W., Wu, M.C., Feng, G.S. and Wang, H.Y. (2012) p28(GANK) Prevents Degradation of Oct4 and Promotes Expansion of Tumor-Initiating Cells in Hepatocarcinogenesis. Gastroenterology, 142, 1547-1558. http://dx.doi.org/10.1053/j.gastro.2012.02.042

[44] Atlasi, Y., Mowla, S.J., Ziaee, S.A. and Bahrami, A.R. (2007) OCT-4, an Embryonic Stem Cell Marker, Is Highly Expressed in Bladder Cancer. International Journal of Cancer, 120, 1598-1602.

[45] Cheng, L., Sung, M.T., Cossu-Rocca, P., Jones, T.D., Maclennan, G.T., De Jong, J., Lopez-Beltran, A., Montironi, R. and Looijenga, L.H. (2007) OCT4: Biological Functions and Clinical Applications as a Marker of Germ Cell Neoplasia. The Journal of pathology, 211, 1-9.

[46] Wang, L., Guo, Z.Y., Zhang, R., Xin, B., Chen, R., Zhao, J., Wang, T., Wen, W.H., Jia, L.T., Yao, L.B. and Yang, A.G. (2013) Pseudogene OCT4-pg4 Functions as a Natural Micro RNA Sponge to Regulate OCT4 Expression by Competing for miR-145 in Hepatocellular Carcinoma. Carcinogenesis, 34, 1773-1781. http://dx.doi.org/10.1093/carcin/bgt139

[47] Jemal, A., Bray, F., Center, M.M., Ferlay, J., Ward, E. and Forman, D. (2011) Global Cancer Statistics. CA: A Cancer 
Journal for Clinicians, 61, 69-90. http://dx.doi.org/10.3322/caac.20107

[48] Sundaram, P., Hultine, S., Smith, L.M., Dews, M., Fox, J.L., Biyashev, D., Schelter, J.M., Huang, Q., Clear, M.A., Volpert, O.V. and Thomas-Tikhonenko, A. (2011) p53-Responsive miR-194 Inhibits Thrombospondin-1 and Promotes Angiogenesis in Colon Cancers. Cancer Research, 71, 7490-7501. http://dx.doi.org/10.1158/0008-5472.CAN-11-1124

[49] Okamoto, K., Ishiguro, T., Midorikawa, Y., Ohata, H., Izumiya, M., Tsuchiya, N., Sato, A., Sakai, H. and Nakagama, H. (2012) miR-493 Induction during Carcinogenesis Blocks Metastatic Settlement of Colon Cancer Cells in Liver. The EMBO Journal, 31, 1752-1763. http://dx.doi.org/10.1038/emboj.2012.25

[50] Luo, H., Zou, J., Dong, Z., Zeng, Q., Wu, D. and Liu, L. (2012) Up-Regulated miR-17 Promotes Cell Proliferation, Tumour Growth and Cell Cycle Progression by Targeting the RND3 Tumour Suppressor Gene in Colorectal Carcinoma. Biochemical Journal, 442, 311-321. http://dx.doi.org/10.1042/BJ20111517

[51] Almeida, M.I., Nicoloso, M.S., Zeng, L., Ivan, C., Spizzo, R., Gafa, R., Xiao, L., Zhang, X., Vannini, I., Fanini, F., Fabbri, M., Lanza, G., Reis, R.M., Zweidler-Mckay, P.A. and Calin, G.A. (2012) Strand-Specific miR-28-5p and miR-28-3p Have Distinct Effects in Colorectal Cancer Cells. Gastroenterology, 142, 886-896. http://dx.doi.org/10.1053/j.gastro.2011.12.047 